\title{
Human cancer is transmitted via genome
}

\author{
Sergey N. Rumyantsev \\ Department of Evolutionary Immunology, Andent, Inc., Jersey City, USA \\ Email: rumyan1@yahoo.com
}

Received 22 January 2013; revised 25 February 2013; accepted 3 March 2013

\begin{abstract}
Recent discredit of "somatic mutation" hypotheses forced the need of new paradigms about the nature of human cancer. The present article is devoted to further development of one such paradigm: the hypothesis of invasive parasitic nature, origin, evolution, pathogenesis and transmission of human cancer. Development was performed by supplementing and supporting the hypothesis by data which could not be applied before. The supplementation included integrative reconsidering, and reinterpretation of the make-ups, traits and processes existing not only in human cancer but also in animal cancers. Special attention has been focused on xenogamous intrusion of carcinogenic traits in the genome of a host. It was evidenced that human cancer possesses the same set of traits characteristic of transmissible animal cancer. In contrast to animal cancer formed of solitary cell lineage, human cancer consists of a couple of lineages constructed under different genetic regulations and performed different structural and physiological functions. The diversity of cancer composition remains stable over sequential propagation. The subsistence of human cancer regularly includes obligatory rotation alternation of its successive forms including genomic, gametic, zygotic, micro-population and tumorous ones. Human cancer possesses its own biological watch and the ability to gobble its victim, transmit via the intrusion of the genome, perform intercommunications within the tumor components and between the dispersed subunits of cancer.
\end{abstract}

Keywords: Cancer genome; Cancer immunology;

Cancerous Parasitism; Genomic intrusion;

Genomeintruder Malevolent; Hereditary Immunity;

Heterozygosity; Regulator-Receptor System;

Self-Reproduction

\section{INTRODUCTION}

For many decades, human cancer, unlike various kinds of animal cancer, was unconditionally considered as absolutely non-transmissible. This hypothetical statement was based on an over 80-year-old hypothesis about the origins of cancers from an individual somatic mutation of individual cells [1] that was supported by a plethora of medical articles, monographs, dictionaries, encyclopedias and websites.

Recently, this unanimity has been disrupted by several publications [2-5] that propose a new paradigm of an invasive parasitic nature, transmission, origin, evolution, and pathogenesis of human cancer. This new paradigm radically changes the set of knowledge about fundamental genetic and physiological mechanisms that underlie cancer's development and progression. In contrast to the hypothesis of somatic mutations, the main focus of the new paradigm is on the genomic roots of cancer, namely on carcinogenic transformations of personal genomes over xenogamous crossbreeding.

Similar to the concept of the genomic roots of cancer [5], a passing remark in one recent review noted that it becomes entirely appropriate to describe cancer as a disease of the genome. It was also accentuated that specific inherited or "germline" genomic alterations can be used to confer the cancer development [6]. A more recent study ( $>12.2$ million individuals, including $>1.1$ million cases of first primary cancer) has shown the existence of a genomic (inherent, familial) component in the development of many cancers-colorectal, lung, breast, prostate, and urinary bladder cancer and melanoma, skin squamous cell carcinoma [7].

In the present article, various details of the new paradigm were developed primarily by supplementing and supporting its core principles with some very important data that had not been previously considered. The supplementation includes mainly the results of integrative reconsidering, systematization and reinterpretation of both the known and recent data concerning the ways of transmission, immunology, genetics, pathogenesis and clinical exposures of natural cancer in not only humans but also in animals. Such an approach has never been exploited in investigations of the matter.

\section{MATERIALS AND METHODS}

The present article is devoted to the development of the new paradigm by supplementing its core principles with 
data which has not been considered before. The supplementation was performed by multidisciplinary reconsidering, systematization, integration and reinterpretation of both known and recent data concerning the ways of transmission, immunology, genetics, pathogenesis and clinical exposures of natural cancer existing among both human and animals.

\section{RESULTS AND DISCUSSION}

\subsection{Origin and Transmissibility of Animal Cancer}

The existence of contagious cancer is well known among dogs and other canines, [8] Tasmanian devils, [9] Syrian hamster, $[10,11]$ sea lions (genital carcinoma), sea turtles (fibropapillomatosis), beluga whales, bottom-dwelling fish [12,13] and so on.

Venereal cancer of dogs is usually transmitted when dogs mate, resulting in the direct physical intrusion of viable cancerous matter from an afflicted dog to a healthy one. The intrusion may also be realized through licking, biting and sniffing tumor-affected areas. The tumor may also be transplanted from site to site and dog to dog by direct contact with the tumorous mass [14]. The tumor masses are themselves the infectious agents. This is considered an infectious disease of dogs caused by a pathogenic lineage of cancerous cells that live as a unicellular pathogen [15].

This kind of cancer is an allograft that is transmitted between individuals without immune recognition of the cancerous intruder as "non-self” $[9,14]$.

All tumor cells of this type of cancer share an extremely similar genetic code, often, if not always, unrelated to the DNA of their host. Genetic data strongly indicate that the tumors do not arise from separate cancerous transformation in individual animals $[15,16]$.

Cancer of Tasmanian devils afflicts a marsupial carnivore endemic to the island of Tasmania. It occurs equally in male and female devils [17]. Transmission is achieved as devils frequently bite one another on the mouth during mating or while fighting for territory [18]. Any case of transmissible cancer of this kind is induced by physical intrusion of viable tumor cells into a susceptible organism in the form of an allograft not recognized immediately by the immunogenic system as non-self. The growths were essentially identical to one another but otherwise genetically distinct from the devils themselves. Genetic analysis reveals the parasitic origin of Tasmanian contagious cancer [9]. The malignancy does not have the same DNA as the animals they are killing.

Contagious sarcoma of the Syrian hamster is able to afflict various individuals in any observed populations [19]. The spontaneously transplantable tumor can spread from an affected to a healthy hamster by direct physical contact as well as by feed of tumor material [20]. This cancer is an allograft that is transmitted between individuals without evoking instant immune response to alloantigens. There are a wide range of individual responses, from no reaction to low or relatively strong responses because in these cases, tumor cells grew in the hosts of various genetic similarities [21].

The tumors appear naturally in the vascular and lymphatic systems of Syrian hamsters $[10,11]$. This is a spontaneously transplantable sarcoma with leukemic manifestations. The transfer of these circulating cells from tumor-bearing hamsters to hamsters without tumors leads to the transmission of the next tumor [11]. According to chromosome studies [10,11], the cells of all tested cancerous animals had identical, very consistent and highly specific tumor karyotype differing from the normal pattern for hamster cells.

The analyze allows us to conclude that each of most discovered transmissible tumors exploits a relevant animal species (dogs, Tasmanian devils or Syrian hamsters) and thus has its unique adaptations to the host. The transmission of animal cancer is realized mainly by natural sexual relations intrinsic for relevant host species. Furthermore, all of these animal cancers have a common etiology: they are transmitted by the physical transfer of viable cancerous cells. The development of individual cancer is initiated in animals by the intrusion into the afflicted body of a deviant cell clone (or clones), inherently immune to normal physiological regulators of cell growth and tissue formation [5].

Animal cancers are also characterized by genetic dissimilarity between cancerous cells and the animals they invade. In other words, any transmissible animal cancer is a result of the intrusion of genetically dissimilar viable somatic material and its subsequent self-reproduction in the invaded living body. The dissimilarity is revealed in the structural and functional traits of the animal's cancer cells. he ability of cancerous cells to perform uncontrollable self-reproduction is the first of these traits and is evidence of the structural immunity of the cells against natural regulation of cell dividing.

\subsection{Transmissibility of Human Cancer}

3.2.1. New Updates to Genomic Ties of Human Cancer Before the paradigm of the parasitic nature of human cancer was developed [4,5], there were no known naturally occurring ways for the transmission and spread of cancer in humans. Even the very thought of cancerous invasion between peoples did not correlate with the prevailing hypothesis of a stochastic origin of any cancer out of somatic mutation of a single cell.

There were only rare reports of artificial cancer transmission between humans by an accidental transfer of cancer cells through organ transplantation or during 
surgical procedures as well as the problematic transfer of cancer cells from mother or co-twin via placenta. Only $0.04 \%$ of organ transplant recipients contract cancer from the donor organ. Furthermore, the survival of transplanted cancers in healthy humans was exceedingly rare and documented in only a handful of cases. Genetic immunity probably prevented such cancers from taking hold [5].

Friends, family members and caregivers of cancer patients need not be unduly concerned with the remote possibility of "catching cancer" [13] via direct physical contact with cancer carriers. Human cancer was not generally considered a transmissible disease. Meanwhile, in the middle of the $20^{\text {th }}$ century, cancer overtook many infectious diseases as an important human killer. It became one of the biggest threats to global human health. It takes a terrible and growing human toll and its prevalence continues to grow.

Meanwhile, like undoubtedly transmissible malignant diseases of animals, human cancer is also characterized by the same set of traits characteristic of malignant growth naturally transmissible among animals. Relevant information of the totality of these traits has recently been summarized and interpreted elsewhere [5].

Undoubted analogies can be seen in the prevalence, clinical exposures, progression of disease, the origin of causative agents, and especially in the genetic deviations characteristic of both animal and human malignancies. Any cancer sustains itself at the expense of substances in the victim's body.

This set of traits includes the abnormal reproduction of some aberrant cells and consequent growth of relevant aberrant tissues in different parts of the afflicted organism. Both animal and human cancers are able to exhaust the life-supporting functions of the invaded body and intoxicate its life supporting organs.

Recent studies [6,22], together with the set of data discussed above, allow us to suppose that like any other multicellular beings, cancer contains a variety of different cells that are under different genetic regulation and possess different behaviors. Observation has shown that cancer consisted of four different clones, with a specific mutational profile and different susceptibilty to chemotherapeutic agents which may decrease clonal heterogeneity. Each clone present at a specific proportion in the tumor cell population [6]. Cancer consists of a couple of functionally heterogeneous cell lineages that vary with respect to their distinctive structural or physiological functions and potentials. The heterogeneity within tumor cell lineages may also determine the differences within the kinds of tumors and their locations. Cancer is able to maintain its structural stability through many generations. The diversity of cancer composition remain stable over its sequential long-term propagation [22].

These means that both animal and human cancers have developed many adaptations that enable these aberrant lineages of mammalian cells to exist as a multicellular parasite $[4,23]$. At the same time, in contrast to animal cancer, the malignant disease of humans does not possess the ability to transfer its living cells from one person to another. Human cancer exploits another way to ensure the maintenance of its own life after its host is exhausted and dies.

\subsubsection{Human Cancer Arises via Intrusion a Victim Genome}

The renewed set of final evidence of the new paradigm [5] allowed confirmation that the descent of human cancer has been predetermined by genome mutations which have created, in evolution, inter-ethnic differences in molecular constitution of intrinsic physiological systems responsible for the regulation of cell dividing and tissue growth. Consequent xenogamous mating between members of such different ethnoses may lead to the intrusion of the descendant's genome with components of deviant genetic information that induce carcinogenesis. The descent and consequent subsistence of human cancer regularly includes obligatory alternation of its successive forms (Table 1). The development of individual cancer is initi ated by the appearance of a deviant cell clone inherently immune to autochthonous regulators of cell growth and tissue formation [5]. The cells are able to grow independent of physiological control of autochthonous cell replication. This clone is foreign (non-self) for the afflicted body with many of its traits.

The deviant cells appear in a human body as the result of genome transformation performed over the heterozygous crossbreeding between parental gametes with partially different (divergent) genotypes [24]. This is a kind of chimerism or cellular mosaicism, the occurrence in an

Table 1. Successive forms of cancer formation and subsistence.

\begin{tabular}{|c|c|c|c|}
\hline \multirow{2}{*}{ Parental ethnoses } & \multicolumn{3}{|c|}{ Successive forms of cancer formation and subsistence } \\
\hline & Genomic forms & forms & Multicellular forms \\
\hline A & $\begin{array}{l}\text { Genome of regular parent Gamete of regular parent } \\
\text { Genome of deviant parent Gamete of deviant parent }\end{array}$ & Carcinogenic zygote & $\begin{array}{l}\text { Micro-populations of Tumors (developed populations } \\
\begin{array}{c}\text { cancerous cells } \\
\text { of cancerous cells) }\end{array}\end{array}$ \\
\hline
\end{tabular}


individual of two or more cell clones of different genome constitutions, derived from different parental individuals $[25,26]$. Such heterozygous mosaicism arises as a result of hybridization between two organisms genetically different in some of the relevant traits. For instance, one of them is constitutionally immune to appropriate physiological regulators whereas its mating partner is constitutionally sensitive to it [2].

The heterozygosity results in the coexistence in the offspring's genome of at least two active allelomorphic genes. Both alleles function dominantly and create two allelic cell clones, whose subpopulations are formed and distributed in the body before postnatal ontogenesis. The heterozygous offspring expresses both alleles equally but in different sizes and in separate locations around the body. Thus, over such xenogamous formation of descendant's zygote, its genome becomes admixed with a block of aberrant, potentially carcinogenic genes. This leads to the intrusion of the offspring's genome with heterozygous genes and the formation in the offspring's body of coexisting cell clones with opposite autochthonous regulators of cell and tissue growth.

The emergence of such a cancerous clone and its dispersion around the body in the form of one or more discrete micro-populations is performed before postnatal ontogeny in the manner used to dispose of other embryonic tissues and organs. That is why, despite the genetic and phenetic dissimilarity of human cancer with its host, the lymphatic system of individual adaptive immunity does not recognize the deposited cancer cells as foreign and does not destroy them. After the end of their disposition, the subpopulations continue to reside in their stable places like cell masses of small but different sizes.

Cancerous cell populations subsist on life supporting functions provided by the host. Any individual cancer arises and exists as a result of natural ecological relations between two organisms in which the xenogamous one [the consumer) obtains the stuff and energy for its life at the expense of substances composed of the consumed organism (the victim or host). Cancer is a kind of parasitism. The marauding way of life exploited by populations of cancerous cells is performed mainly by their molecular enzymatic agents, targeted either on the spliting of the host's macromolecules or producing functional inhibition of the host cells.

At a relevant time of a host's life (mainly after 40 years of age), the uncontrollable growth of such microsubpopulations becomes visible in the form of detectable extra cell masses of cancerous tissue, the malignant tumors. The largest of the subpopulations achieves the size of detectable tumor far earlier than the smaller ones, thus forming the first appeared cell mass usually called the "primary" tumor.

The growth of all subpopulations of a cancerous clone is controlled by their own united physiological mechanism which maintains the whole structure of cancer within a genetically predetermined size. The destruction of one or more tumors boosts the growth of other subunits of the clone. Various evidence of this intriguing phenomenon has recently been summarized and interpreted elsewhere [5].

Human cancer possesses its own schedule (the program of ontogenesis) as well as the abilities of communication and physiological synchronization between its intra-host subunits. The existence of these intrinsic traits has been initially estimated $[4,5,23,24]$ with detailed presentation and discussion of the evidence. Later, it has been experimentally shown that the spread of melanoma in the mouse model correlated with the eventual progress of the disease in human patients. Conversely, melanomas that did not progress after surgical removal of the primary tumors from patients also developed slowly or inefficiently in animals, even after repeated passages of tumor cells through several generations of mice. This finding also demonstrated that the key factors that regulate the rate of cancer and mode of development are intrinsic to the invading cancerous matter [27].

Thus human cancer possesses a set of constitutional adaptive traits that could be thought to be a result of evolution over many hundreds of millennia. The date of its initiation could be referred, for instance, to regular hybridization and exchange of genes between mutual ancestors of chimps and humans that may have occurred over a few million years [28] as well as to the epoch of xenogamous intercourse of European Homo sapiens with Homo neandertalensis. The last gene flow from Neanderthals (or their relatives) into Europeans likely occurred 37,000 - 86,000 years ago, and most likely 47,000 65,000 years ago [29].

\subsubsection{Human Cancer Transmits via Intrusion of Genome}

According to the new paradigm, cancer belongs to the group of invasive diseases whose subsistence depends on regular transmission of the causative agent from one victim's body to another. Infections and parasitic invasions belong to the group too. Like any other contagious disease, cancer arises and exists as a result of natural ecological relations between two species in which the contagious species (the consumer) obtains the matters and energy for its life, reproduction and subsequent transmission at the expense of substances contained in the victim. These actions exhaust the lifeblood out of afflicted body and thus restrict its vitality, provoking the state of disease and a loss of victim viability. Once filled with cancerous agents, the body of the affected victim serves as a source of contagion into new victims.

The transmission of invasive agents inside the next 
victim's body is mainly carried out by means of the victim's ecological communications, through which the regular physiological functions are provided; for example, through feeding (as an alimentary transmission), breathing (respiratory transmission), and self-reproduction (sexual transmission). Human cancer transmits via sexual intercourse.

The carrier of human cancer is characterized by a complex of traits necessary for providing the host's ability to transmit deviant genomes into relevant gametes, execute multifold acts of fertilization and breed descendants to the stage that is usually called complete maturity. The absence of any of the abilities sharply diminishes the chances of the cancerous genome to prolong its life in the genomes of descendent generations.

The growth of human cancer results in its initial micro-populations becoming visible in the form of tumors mainly after 40 years of age. This may mean human cancer possesses its own schedule of life (the program of ontogenesis) different of those belonging to its victim. Natural selection favors those cancerous cell lineages whose schedule of life did not restrict the reproductive, i.e. transmissive, function of the afflicted person nor its care for its offspring up to the reproductive (transmissive) stage. Saving its own life via self-reproduction is an extraordinarily important function of any form of living matter. Human cancer performs this function very successfully by maintaining long-term propagation.

Human cancer, the recently discovered kind of parasitic beings [5], also developed all relevant adaptations including the ability to circulate between people. This is a form of life that was unidentified and unclassified before. This parasitic invader should belong to a new type and class of multicellular parasites, Species-Genomeintruder malevolent (G. malevolent). The species developed in evolution not only banal but also unique adaptations to its modus vivendi.

During the formation of intruded zygote, the deviant components of xenogamous genetic code appear to be included in the formed united genetic code. Since the components continue to exist in it and function over the creation of intruded cells with their plethora of both banal and unique traits. Being implanted in the genome of its current host, the genome of human cancer ensured that it was reproduced in the genomes of children via self-reproduction of the cancer-carrying parent. Like any other components of genetic code, they are able to be reproduced in the descendant genomes and thus multiplied and dispersed between people.

\subsection{Conclusions}

The subsistence of cancerous cells and tumor masses is provided at the expense of both the structures (proteins, lipids, saccharides) and functions (the supply of oxygen, nutritive substances and means for reproduction) of the invaded organism. Independent of the host species, any transplantable animal cancer is able to suck the lifeblood out of the afflicted body. This is a kind of marauding parasitism performed by the invaded cells of dissimilar animal origin. In any of the species discussed above, cancer develops without immediate immune recognition of the invading cancerous matter.

Cancer, regardless of the species in which it occurs, is the uncontrolled growth of tissue abnormal and "nonself" for the afflicted organism. However, there are substantial differences in cancer transmission and pathogenesis dependent on the species. The biggest contrasts in the modes of transmission were revealed between contagious cancers of animals and humans.

The current pandemic spread of human cancer has been brought about by the growing expansion of interethnic admixture favored by growing industrialization, urbanization, globalization, and migration. The currently observed increasing incidence of the disease has been induced by the intensification of xenogamous genetic admixture within ethnically mixed populations. These new notions provide the framework and some initial landmarks for the location of genomic ties and roots of cancer origin and should encourage the appearance of new research ideas and proposals for cancer prevention and therapy. There remains much to be learned about this extraordinarily unique and extremely complex disease. According to the xenogamous paradigm, the search for a coveted clue to the genomic roots of cancer should be oriented on the discovery of structural and functional differences between the genomes of cancerous and normal cells.

The initial overall prevention of cancer could be started by the voluntary restriction of xenogamous fertilization as well by the launching of noncancerous genealogies. The risk of cancer development for our children should be at the core of prophylactic doctrine. "Will our children develop cancer?” This tough question should be asked by each couple before they marry. The genealogies of expectant moms and dads must be discovered for the absence of cancer disease among any of their accessible ascendants and genetic relatives. This kind of protective parenting is now on its way to becoming a mainstream medical testing at first by routine genetic methods but finally or in special cases by whole genome sequencing. It is time now to start searching the genomes of every groom and bride in order to assess risk of producing carcinogenic combinations in the genomes of their descendants. Appropriate genomic tests must be performed before conception. The results can provide early warnings about the deadliest and most debilitating disease that may not strike until adulthood. Those warnings can help 
people either to make rationale decisions about their marital plans or at least to be prepared to enable timely treatment and elaborate plans about long-term care.

\section{REFERENCES}

[1] Bauer, K.H. (1928) Mutationstheorie der geschswulstEntstehung. Springer, Berlin. doi:10.1007/978-3-642-99670-2

[2] Rumyantsev, S.N. (2008) Hereditary immunity: Fundamental principles and exploitation in life study and health care. New York, Nova Biomedical Books.

[3] Rumyantsev, S.N. (2009) The discredit of cancer metastasis. Science Advisory Board. http://www.scienceboard.net/community/perspectives.22 $\underline{\text { 7.html }}$

[4] Rumyantsev, S.N. (2009) The uniqueness and ordinaryness of cancer origin and pathogenesis: New epidemiological, clinical and preventive perspectives. Journal of Clinical Medicine Research, 1, 32-36.

[5] Rumyantsev, S.N. (2012) Toward the genomic roots of cancer. Journal of Medicine and Medical Sciences, 3, 638-659.

[6] Mardis, E.R. (2012) Genome sequencing and cancer. Current Opinion in Genetics \& Development, 22, 245250. doi:10.1016/j.gde.2012.03.005

[7] Kharazmi, E., Fallah, M., Sundquist, K. and Hemminki, K. (2012) Familial risk of early and late onset cancer: Nationwide prospective cohort study. BMJ, 345, e8076. doi:10.1136/bmj.e8076

[8] Rebbeck, C.A., Thomas, R., Breen, M., Leroi, A.M. and Burt, A. (2009) Origins and evolution of a transmissible cancer. Evolution, 63, 2340-2349. doi:10.1111/j.1558-5646.2009.00724.x

[9] Murchison, E.P. (2008) Clonally transmissible cancers in dogs and Tasmanian devils. Oncogene, 27, S19-S30. doi:10.1038/onc.2009.350

[10] Banfield, W.G., Woke, P.A., Mackay, C.M. and Cooper, H.L. (1965) Mosquito transmission of a reticulum cell sarcoma of hamsters. Science, 148, 1239-1240. doi:10.1126/science.148.3674.1239

[11] Cooper, H.L., Mackay, C.M. and Banfield, W.G. (1964) Chromosome studies of a contagious reticulum cell sarcoma of the Syrian hamster. Journal of the National Cancer Institute, 33, 691-706.

[12] McAloose, D. and Newton, A.L. (2009) Wildlife cancer: A conservation perspective. Nature Reviews Cancer, 9, 517-526. doi:10.1038/nrc2665

[13] Welsh, J.S. (2011) Contagious cancer. Oncologist, 16, 14. doi:10.1634/theoncologist.2010-0301

[14] Mello Martins, M.I., de Souza, F.F. and Gobello, C. (2005) Canine transmissible venereal tumor: Etiology, pathology, diagnosis and treatment. Recent Advances in Small Animal Reproduction.

[15] Murgia C, Pritchard, J.K., Kim, S.Y., Fassati, A. and Weiss, R.A. (2006) Clonal Origin and evolution of a transmissible cancer. Cell, 126, 477-487. doi:10.1016/j.cell.2006.05.051

[16] Dingli, D. and Nowak, M.A. (2006) Cancer biology: Infectious tumour cells. Nature, 443, 35-36. doi:10.1038/443035a

[17] Hawkins, C.E., Baars, C., Hesterman, H., Hocking, G.J., Jones, M.E., Lazenby, B., Mann, D., Mooney, N., Pemberton, D. and Pyecroft, S. (2006) Emerging disease and population decline of an island endemic, the Tasmanian devil Sarcophilus harrisii. Biological Conservation, 131, 307-324. doi:10.1016/j.biocon.2006.04.010

[18] Pearse, A.M. and Swift, K. (2006) Allograft theory: Transmission of devil facial-tumour disease. Nature, 439, 549. doi:10.1038/439549a

[19] Pour, P., Mohr, U., Althoff, J., Cardesa, A. and Kmoch, N. (1976) Spontaneous tumors and common diseases in two colonies of Syrian hamsters. IV. vascular and lymphatic systems and lesions of other sites. Journal of the National Cancer Institute, 56, 949-961.

[20] Brindley, D.C. and Banfield, W.G. (1961) A contagious tumor of the hamster. Journal of the National Cancer Institute, 26, 949-957.

[21] Kreiss, A., Cheng, Y., Kimble, F., Wells, B., Donovan, S., Belov, K. and Woods, G.M. (2011) Allorecognition in the Tasmanian devil (Sarcophilus harrisii), an endangered marsupial species with limited genetic diversity. PLoS One, 6, e22402. doi:10.1371/journal.pone.0022402

[22] Kreso, A., O’Brien, C.A., van Galen, P., Gan, O., Notta, F., Brown, A.M.K., Ng, K., Ma, J., Wienholds, E., Pollett, A., Gallinger, S., McPherson, J., Mullighan, C.J., Shibata, D. and Dick, J.E. (2012) Variable clonal repopulation dynamics influence chemotherapy response in colorectal cancer. Science, 338, 6113.

[23] Rumyantsev, S.N. (2010) Hypothesis: Towards the origin of cancer epidemics and pathogenesis. Journal of Carcinogenesis, 9, 1-7.

[24] Rumyantsev, S.N. (2011) Functions of hereditary immunity and xenogamy in cancer origin and pandemic spread. Open Journal of Immunology, 1, 27-40. doi:10.4236/oji.2011.12004

[25] Bonnicksen, A.L. (2009) Chimeras, hybrids, and interspecies research: Politics and policymaking. Georgetown University Press, Washington.

[26] McLaren, A. (1976) Mammalian chimaeras. Cambridge University Press, Cambridge.

[27] Quintana, E., Piskounova, E., Shackleton, M., Weinberg, D., Eskiocak, U., Fullen, D.R., Johnson, T.M. and Morrison, S.J. (2012) Human melanoma metastasis in NSG mice correlates with clinical outcome in patients. Science Translational Medicine, 7, 159.

[28] Patterson, N., Richter, D.J., Gnerre, S., et al. (2006) Genetic evidence for complex speciation of humans and chimpanzees. Nature, 441, 1103-1108. doi:10.1038/nature04789

[29] Sankararaman, S., Patterson, N., Paabo, S. and Reich, D. (2012) The Date of Interbreeding between Neandertals and Modern Humans. PLoS Genetics, 8, e1002947. doi:10.1371/journal.pgen.1002947 